\title{
The Role of Thalamic Inputs in Surround Receptive Fields of Barrel Neurons
}

\author{
Ernest E. Kwegyir-Afful, ${ }^{1}$ Randy M. Bruno, ${ }^{2,3}$ Daniel J. Simons, ${ }^{2}$ and Asaf Keller ${ }^{1}$ \\ ${ }^{1}$ Program in Neuroscience and Department of Anatomy and Neurobiology, University of Maryland School of Medicine, Baltimore, Maryland 21201, \\ ${ }^{2}$ Department of Neurobiology, University of Pittsburgh School of Medicine, Pittsburgh, Pennsylvania 15261, and ${ }^{3}$ Max Planck Institute, Department of \\ Cellular Physiology, Heidelberg 69120, Germany
}

Controversy exists regarding the relative roles of thalamic versus intracortical inputs in shaping the response properties of cortical neurons. In the whisker-barrel system, this controversy centers on the mechanisms determining the receptive fields of layer IV (barrel) neurons. Whereas principal whisker-evoked responses are determined by thalamic inputs, the mechanisms responsible for adjacent whisker (AW) responses are in dispute. Here, we took advantage of the fact that lesions of the spinal trigeminal nucleus interpolaris $(\mathrm{SpVi})$ significantly reduce the receptive field size of neurons in the ventroposterior thalamus. We reasoned that if AW responses are established by these thalamic inputs, brainstem lesions would significantly reduce the receptive field sizes of barrel neurons. We obtained extracellular single unit recordings from barrel neurons in response to whisker deflections from control rats and from rats that sustained SpVi lesions. After SpVi lesions, the receptive field of both excitatory and inhibitory barrel neurons decreased significantly in size, whereas offset/onset response ratios increased. Response magnitude decreased only for inhibitory neurons. All of these findings are consistent with the hypothesis that AW responses are determined primarily by direct thalamic inputs and not by intracortical interactions.

Key words: thalamus; trigeminal; vibrissa (whisker); rat; thalamocortical and corticofugal projections; somatosensory cortex

\section{Introduction}

Cortical neurons respond preferentially to activation of the center of their peripheral receptive fields, whereas stimulation of the penumbra of the receptive field evokes a weaker excitatory response (Mountcastle et al., 1957; Mountcastle and Powell, 1959; Hubel and Wiesel, 1968). Responses to stimulating receptive field centers are generated by direct thalamic inputs (Hubel and Wiesel, 1962; Armstrong-James, 1995; Simons, 1995). However, there is controversy regarding the generation of excitatory responses to stimulating the penumbra, with arguments for both intracortical (Armstrong-James et al., 1991; Fox, 1994; Fox et al., 2003) and thalamocortical (Simons and Carvell, 1989; Goldreich et al., 1999) mechanisms being advanced. A similar controversy surrounds the source of inputs that determine orientation selectivity in visual cortical neurons (for review, see Ferster and Koch, 1987), with some arguing for a purely thalamocortical mechanism (Hubel and Wiesel, 1962; Dalva et al., 1997), whereas others argue for a predominant intracortical mechanism (Wolf et al., 1986; Douglas et al., 1995). Others still have argued for an "eclectic" model in which weak thalamocortical inputs are modified by an intracortical mechanism (Worgotter and Koch, 1991).

Received April 7, 2005; revised May 10, 2005; accepted May 11, 2005

This work was supported by United States Public Health Service/National Institute of Neurological Disorders and Stroke Grants NS-31078 and NS-35360 (A.K.) and NS-19950 (D.J.S.)

Correspondence should be addressed to Dr. Asaf Keller, Department of Anatomy and Neurobiology, University of Maryland School of Medicine, 20 Penn Street, Baltimore, MD 21201. E-mail: akeller@umaryland.edu. DOI:10.1523/JNEUROSCI.1360-05.2005

Copyright $\odot 2005$ Society for Neuroscience $\quad$ 0270-6474/05/255926-09\$15.00/0
The rodent primary somatosensory cortex (SI) provides an ideal system to study receptive field formation. Layer IV of the rodent SI contains cellular aggregates called barrels, which are anatomical correlates of the mystacial vibrissas (whiskers) (Woolsey and Van der Loos, 1970). Cells in each barrel respond preferentially to one principal whisker (PW) (Simons, 1985) and, more weakly, to one to four adjacent whiskers (AWs) (Armstrong-James and Fox, 1987). It is agreed that PW responses are established by direct thalamocortical inputs to layer IV neurons (Simons and Carvell, 1989; Armstrong-James et al., 1991). There is less agreement on the source of AW responses. Some propose that the whisker-trigeminal system is a labeled-line pathway, in which subcortical structures relay to the cortex only PW inputs and that AW responses are therefore synthesized by intracortical interactions (Armstrong-James and Callahan, 1991; Armstrong-James et al., 1991; Fox et al., 2003). Others argue that $\mathrm{AW}$ responses in layer IV are independent of intracortical interactions, reflecting direct thalamic inputs that relay both PW and AW inputs (Simons and Carvell, 1989; Goldreich et al., 1999).

One approach to address this controversy is to manipulate the receptive field of thalamic neurons while leaving intracortical mechanisms intact. After lesions of the trigeminal nucleus interpolaris $(\mathrm{SpVi})$, thalamocortical neurons in the ventral posterior medial (VPM) nucleus respond almost exclusively to their PW (Friedberg et al., 2004; Timofeeva et al., 2004). As a result, layer IV neurons in barrel cortex receive, from thalamic afferents, inputs from only PWs. We reasoned that if AW responses were generated intracortically, they would be mostly resistant to this 
manipulation. In contrast, if AW responses were generated subcortically, barrel neurons responses to AW stimuli would be suppressed by this manipulation. Our results are consistent with the second alternative, supporting the hypothesis that direct thalamic inputs are responsible for both the PW and AW responses of barrel neurons. Some of these findings appeared previously in abstract form (Kwegyir-Afful et al., 2004).

\section{Materials and Methods}

Surgical procedures. Twenty-three female Sprague Dawley rats weighing $220-280$ g were used in this study. All procedures strictly adhered to institutional and federal guidelines. The surgical procedures used here have been described previously (Simons and Carvell, 1989; KwegyirAfful and Keller, 2004). Briefly, surgical procedures were done under halothane anesthesia (3\%) and infusion of local anesthetics at surgical sites. A craniotomy was performed over the right primary somatosensory cortex and the contralateral SpVi. In five of the rats, a craniotomy was made over the right VPM instead of SI. In all cases, the dura was removed before recording. Following stereotaxic coordinates specified by Timofeeva et al. (2004), lesions were made in SpVi of six rats, and midline brainstem lesions were made in three other rats. Lesions were made with 160- $\mu \mathrm{m}$-tip-diameter tungsten electrodes with $500 \mu \mathrm{m}$ of the tip exposed. For each type of lesion, the electrode was advanced to the base of the brain and retracted for $500 \mu \mathrm{m}$, and four lesions were made at 500 $\mu \mathrm{m}$ intervals. For SpVi nuclear lesions, a $3 \mathrm{~mA} \mathrm{DC}$ current pulse was passed for $4 \mathrm{~s}$ in all four locations. Midline brainstem lesions were made by passing $2 \mathrm{~mA} \mathrm{DC}$ currents for $3 \mathrm{~s}$ at the four depths described above. After the lesions, rats were removed from the stereotaxic and headmounted by means of a post affixed to the skull, as described previously (Kwegyir-Afful and Keller, 2004).

After completion of surgical procedures, halothane anesthesia was terminated, and the rats were infused intravenously with fentanyl (10 $\left.\mu \mathrm{g} \cdot \mathrm{kg}^{-1} \cdot \mathrm{h}^{-1}\right)$ and pancuronium bromide $\left(1.5 \mathrm{mg} \cdot \mathrm{kg}^{-1} \cdot \mathrm{h}^{-1}\right)$ for the duration of the experiment. Blood pressure, heart rate, and electrocorticograms were monitored throughout the experiment to ensure that the animal was in no pain or distress. Body temperature was maintained at $37^{\circ} \mathrm{C}$ with a servo-controlled heating blanket.

Recording and stimulation. Extracellular unit recordings were obtained with quartz-insulated platinum electrodes (2-4 M 2 ) $2 \mathrm{~h}$ after brainstem lesions were made. Electrodes were advanced perpendicular to the cortical surface using a hydraulic manipulator (Narishige, Tokyo, Japan). Whiskers on the contralateral face were continually stimulated during electrode penetrations to detect units with low or no spontaneous activity. Waveforms recorded from well isolated units were digitized through a Plexon (Dallas, TX) data acquisition system at $40 \mathrm{kHz}$. Units were isolated off-line with a Plexon Offline Sorter, and autocorrelograms were generated with Neuroexplorer (Littleton, MA) software to confirm that recordings were obtained from single units.

Selected recording sites were marked with electrolytic lesions ( $5 \mu \mathrm{A}$ for $10 \mathrm{~s})$. After the experiment, animals were anesthetized deeply with sodium pentobarbital $(60 \mathrm{mg} / \mathrm{kg})$ and perfused transcardially with buffered saline followed by $4 \%$ buffered paraformaldehyde. Recording and lesion sites were identified in cytochrome oxidase- or Nissl-stained tangential sections (Fig. 1).

Whisker stimulation. Receptive fields were initially determined by manually deflecting individual whiskers. Whiskers evoking detectable responses were then individually attached, $10 \mathrm{~mm}$ from their base, to a computer-controlled piezoelectric stimulator that can be deflected in eight different directions. Ramp-and-hold stimuli, $200 \mathrm{~ms}$ in duration and having an onset and offset velocity of $102 \mathrm{~mm} / \mathrm{s}$, were applied at $1 \mathrm{~Hz}$. To reduce mechanical ringing, the trapezoid ramp-and-hold waveforms were filtered with a Bessel filter. The peak onset and offset velocity were measured as the slope of the linear portion of the deflection ramp. The stimulator was calibrated with a photodiode device. Individual whiskers were deflected in one of eight directions (in $45^{\circ}$ increments), delivered randomly for a total of 15 stimuli per deflection angle.

Data analysis. Time stamps of well isolated units and of stimulus triggers were exported to Matlab (MathWorks, Natick, MA) for analyses
A

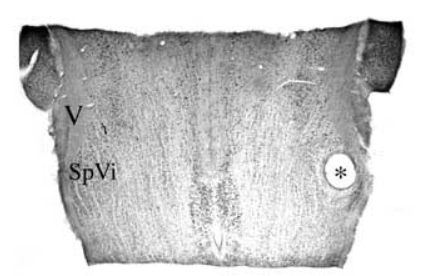

B

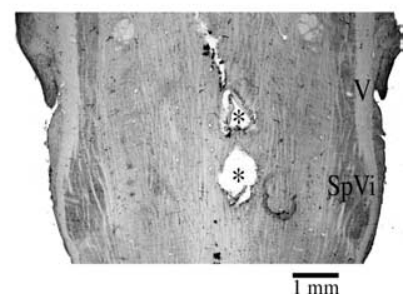

Figure 1. A photomicrograph of a Nissl-stained horizontal section through the brainstem showing a lesion in $\mathrm{SpVi}(\boldsymbol{A})$ and a cytochrome oxidase-stained section showing a midline lesion (B). Asterisks mark lesion sites. V, Trigeminal tract.

using custom-written software. Peristimulus time histograms (PSTHs; 1 ms bins) were constructed from these time stamps. PSTHs were constructed from responses to stimulation of a whisker at all eight angles. Significant stimulus-evoked responses were defined as PSTH bins with a response magnitude that significantly exceeded ( $99 \%$ confidence interval) spontaneous activity levels, computed from a $200 \mathrm{~ms}$ period preceding the stimuli.

Statistical analyses were performed in SPSS (Chicago, IL) and Microsoft (Seattle, WA) Excel. Where appropriate, results are displayed using a boxplot to depict the median and distribution of the data. Between-group statistical comparisons were assessed with the nonparametric Mann-Whitney $U$ test. Within-group (individual neuron) comparisons made use of one-tailed Student's $t$ tests. Categorized data were analyzed using a $\chi^{2}$ test. To describe data distributions fully, both means \pm SD and medians are presented.

\section{Results}

Included in the following analysis are 205 well isolated units of which 118 were from control animals and 87 from animals with brainstem lesions (Table 1). Of this total, 132 were identified (see Materials and Methods) to be in layer IV of the barrel cortex (53 in lesioned animals and 79 in control animals) and 28 in layer V. Layer IV neurons were recorded from microdrive depths of $750-$ $1000 \mu \mathrm{m}$, whereas layer V cells were obtained from depths of $1100-1400 \mu \mathrm{m}$. We only report data for layer IV neurons recorded in barrel centers. An additional 19 cells were recorded from VPM of two animals with brainstem lesions and 22 cells from VPM of three control rats. This was done to ascertain that under our recording conditions the reduction in the receptive field sizes of VPM neurons after SpVi lesions is comparable with that reported by Timofeeva et al. (2004).

Using criteria described by Bruno and Simons (2002), we classified cells recorded from SI as regular spike units (RSUs; presumed excitatory cells) and fast spike units (FSUs; presumed inhibitory cells) based on the following criteria: RSUs were defined as units with waveforms that had an initial negativity (N1) lasting $>180 \mu \mathrm{s}$, followed by a positivity (P1) lasting $>400 \mu \mathrm{s}$. FSUs had an $\mathrm{N} 1$ component $\leq 175 \mu$ s and a P1 component $\leq 350 \mu$ s.

Some control FSU data were included in our previously published study (Bruno and Simons, 2002). There were no statistically significant differences between these data and the control FSU data collected specifically for the current study.

We were concerned that as a result of the proximity of the trigeminal tract to $\mathrm{SpVi}$, lesions of the latter may inadvertently affect the trigeminal tract. This could result in changes in response properties that can be attributed to nonspecific effects caused by damage to the trigeminal tract. To address this, in some animals we made midline brainstem lesions that sever crossedascending axons from SpVi (see Materials and Methods). Such parasagittal brainstem transections have been shown to reduce significantly receptive field sizes of neurons in $\mathrm{SpVi}$, principal 
trigeminal nucleus (PrV), and VPM (Timofeeva et al., 2004). In all cases, we compared the results from cells recorded from animals with midline lesions $(n=$ 13) and animals with trigeminal tract lesions $(n=38)$. Results were combined from these two types of lesions when there was no significant difference between the parameters tested. Of the cells recorded from layer IV, 39 RSUs and 14 FSUs were obtained from rats with brainstem lesions, and of the 79 cells recorded from control rats, 35 were RSUs, whereas 44 were FSUs.

We first present receptive field data for VPM neurons and then describe in detail results pertaining to layer IV barrel neurons. Data on layer V cells are presented in a separate paragraph.

\section{VPM neurons}

Receptive field size

Included in the analysis for receptive field size are cells for which both the principal whisker and all four surround whiskers were tested. A cell was considered to have responded to whisker deflections when we observed a significant response (>99\% confidence level) within a $20 \mathrm{~ms}$ time window after whisker deflection.

Figure $2 \mathrm{~A}$ shows sample PSTHs of a control VPM neuron to deflections of the principal whisker (center) and the four adjacent whiskers. This neuron responded to the PW and all four AWs. Figure $2 B$ shows sample PSTHs of a VPM neuron obtained from a rat with a brainstem lesion. The neuron responded robustly to the PW and minimally to one AW (D3). To compare the changes in receptive field size, we constructed a distribution histogram (Fig. 2C) from 22 neurons recorded from the VPM of control rats and 19 neurons recorded from rats with brainstem lesions. In control animals, $63.6 \%$ of VPM neurons had receptive field sizes between three and five with $36.4 \%$ having receptive field sizes between one and two. In contrast, only $21.1 \%$ of neurons in lesioned animals responded to three to five whiskers with $78.9 \%$ having receptive field sizes between one and two. This decrease in receptive field sizes is consistent with results described by Timofeeva et al. (2004).

To compare these changes quantitatively, we computed, for each cell, the mean response magnitude (in spikes per stimulus) of all four AWs and normalized it to the mean response magnitude of the PW to obtain an AW/PW ratio (Minnery and Simons, 2003). Mean AW/PW ratio for VPM neurons from control rats was $0.13 \pm 0.17$ (median, 0.05), which was sig-
Table 1. Effects of brainstem lesions on response properties of barrel neurons

\begin{tabular}{|c|c|c|c|c|}
\hline Cell type/condition & AW/PW & $\begin{array}{l}\text { Response magnitude } \\
\text { (spikes per stimulus) }\end{array}$ & OFF/ON & $\begin{array}{l}\text { ON response } \\
\text { duration (ms) }\end{array}$ \\
\hline \multicolumn{5}{|l|}{ RSUs } \\
\hline Control & $0.21 \pm 0.18^{*}$ & $0.74 \pm 0.62$ & $0.76 \pm 0.32^{*}$ & $34.46 \pm 25.50^{*}$ \\
\hline Lesion & $0.11 \pm 0.12$ & $0.61 \pm 0.55$ & $1.43 \pm 1.83$ & $21.94 \pm 10.96$ \\
\hline Midline & $0.17 \pm 0.15$ & $0.95 \pm 0.69$ & $1.35 \pm 0.51$ & $17.57 \pm 4.65$ \\
\hline Nuclear & $0.11 \pm 0.11$ & $0.50 \pm 0.48$ & $1.49 \pm 2.12$ & $23.12 \pm 11.91$ \\
\hline \multicolumn{5}{|l|}{ FSUs } \\
\hline Control & $0.35 \pm 0.22^{*}$ & $2.68 \pm 1.60^{*}$ & $0.71 \pm 0.29^{* *}$ & $20.82 \pm 8.85$ \\
\hline Lesion & $0.12 \pm 0.11$ & $1.17 \pm 0.69$ & $0.99 \pm 0.41$ & $17.77 \pm 8.15$ \\
\hline Midline & $0.19 \pm 0.12$ & $1.31 \pm 0.38$ & $1.0 \pm 0.49$ & $16.80 \pm 5.54$ \\
\hline Nuclear & $0.07 \pm 0.08$ & $1.08 \pm 0.85$ & $0.98 \pm 0.39$ & $18.38 \pm 9.75$ \\
\hline \multicolumn{5}{|l|}{ Infragranular RSUs } \\
\hline Control & $0.31 \pm 0.22$ & $0.83 \pm 0.63$ & $0.55 \pm 0.30$ & $46.36 \pm 30.17$ \\
\hline Lesion & $0.31 \pm 0.19$ & $2.00 \pm 1.68$ & $0.67 \pm 0.28$ & $28.00 \pm 12.62$ \\
\hline
\end{tabular}

Values are reported as mean $\pm S D$.

${ }^{*} p<0.01$ and ${ }^{* *} p<0.05$, statistically significant differences between control and lesion values.

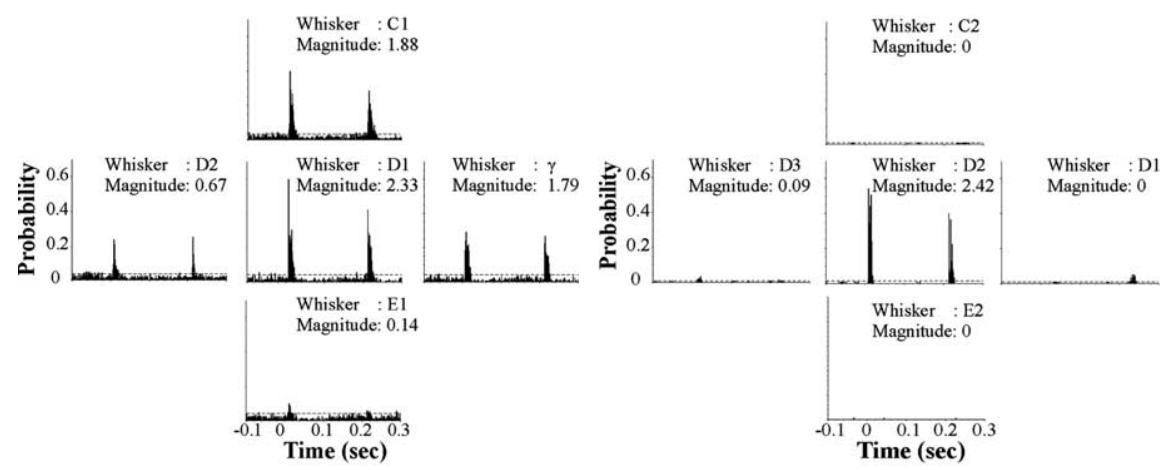

C

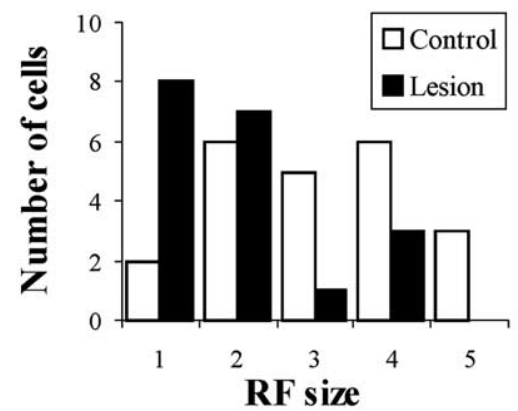

D

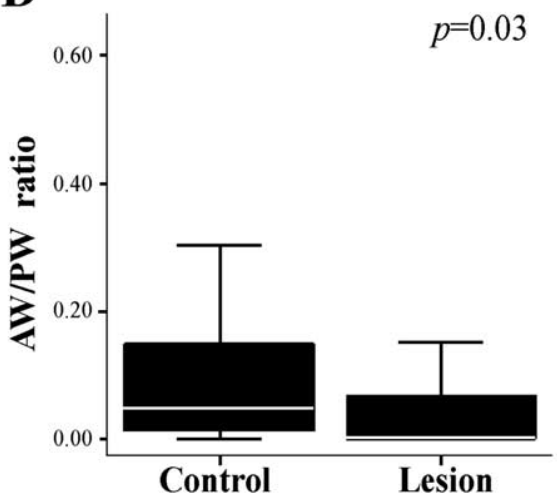

Figure 2. Representative PSTHs showing responses of a control VPM neuron ( $\boldsymbol{A})$ and a VPM neuron from an animal that sustained a brainstem lesion $(\boldsymbol{B})$ to deflection of the principal whisker (center) and four adjacent whiskers. The horizontal dotted line represents the $99 \%$ confidence interval. Stimulus onset is at $t=0$, and stimulus offset at $t=200 \mathrm{~ms}$. The cell depicted in $\boldsymbol{A}$ had a receptive field size of five, whereas that in $\boldsymbol{B}$ was two. $\boldsymbol{C}$, A histogram showing the distribution of receptive field (RF) sizes of VPM neurons in control animals and in animals that sustained a brainstem lesion. The distribution shifts to smaller receptive field sizes after the lesion. D, Boxplots showing the distribution of AW/PW response magnitude ratios of VPM neurons in control animals and in animals with brainstem lesions (Lesion). There is a significant ( $p=0.03$; Mann-Whitney $U$ test) reduction in AW/PW ratios after the lesions. "Whiskers" on boxplots represent data that lay within 1.5 times the interquartile range from the first quartile to the third quartile. 
A B
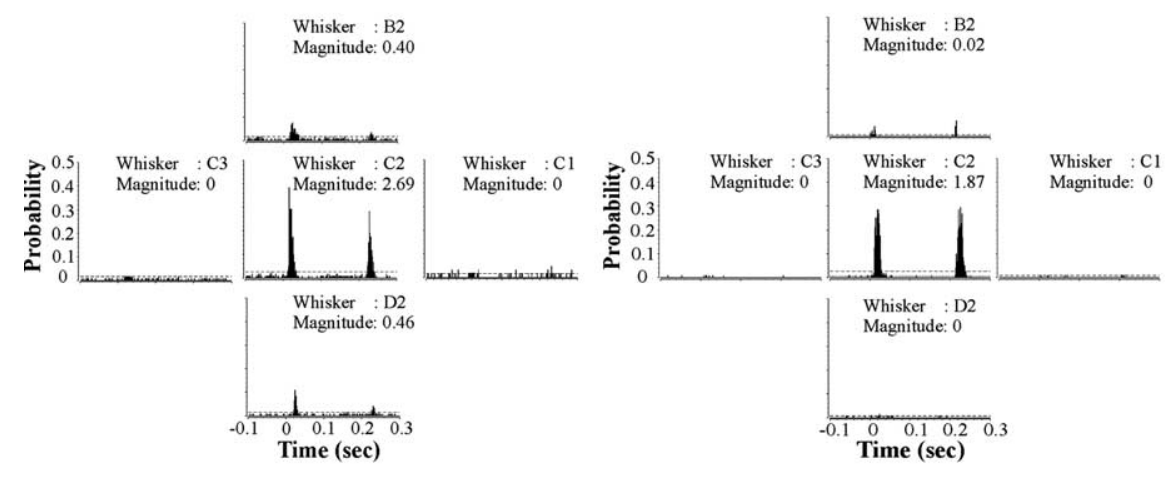

C

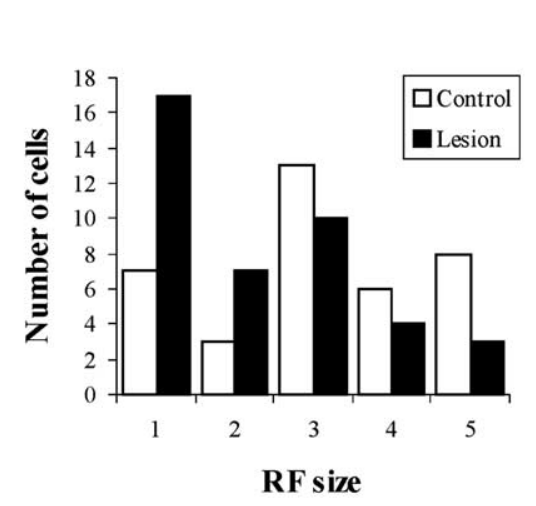

D

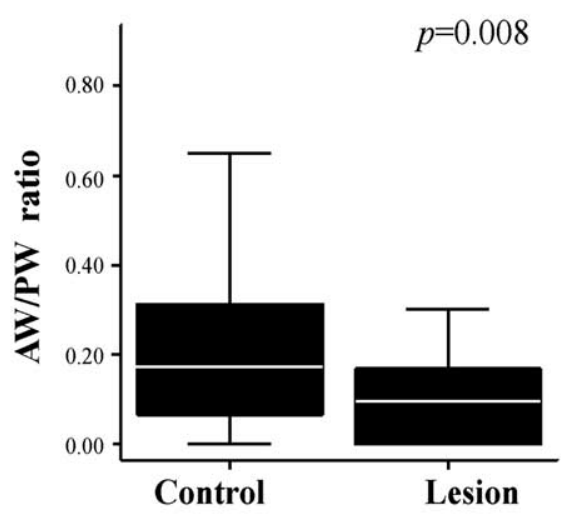

Figure 3. Representative PSTHs showing responses of a control RSU ( $\boldsymbol{A}$ ) and an RSU from an animal that sustained a brainstem lesion ( $\boldsymbol{B}$ ) to deflection of the principal whisker (center) and four adjacent whiskers. The cell depicted in $\boldsymbol{A}$ had a receptive field size of three, whereas that in $\boldsymbol{B}$ was two. $\boldsymbol{C}$, The distribution of receptive field (RF) sizes of RSUs shifts to smaller numbers after the lesion. $\boldsymbol{D}$, Boxplots showing the distribution of AW/PW response magnitude ratios of RSUs in control animals and animals with brainstem lesions. There is a significant ( $p=0.008$; Mann-Whitney $U$ test) reduction in AW/PW ratios after the lesions. "Whiskers" are as described in the legend to Figure 2.

nificantly reduced to $0.06 \pm 0.11$ (median, 0.002 ) (Fig. $2 B$ ) after brainstem lesions ( $p=0.03$; Mann-Whitney $U$ test).

\section{Response kinetics}

Mean PW response magnitudes were similar in control and lesion animals ( $1.28 \pm 0.81$ spikes per stimulus, median, 1.06 vs $1.07 \pm 0.69$ spikes per stimulus, median, $0.89 ; p=0.42$ ) (see Fig. $5 A)$. This finding is in contrast to that of Friedberg et al. (2004), who reported a significant increase in response magnitude of VPM neurons in the absence of SpVi. This discrepancy may be attributable to methodological differences, because Friedberg et al. (2004) performed their recordings 4-7 d after brainstem lesions, a time period sufficiently long for plasticity to occur in VPM (Rhoades et al., 1987).

Brainstem lesions also significantly reduced the spontaneous firing rates of VPM neurons $(p=0.001): 5.4 \pm 6.6 \mathrm{~Hz}$ (median, 3.3) in control animals and $1.7 \pm 2.7 \mathrm{~Hz}$ (median, $0.6)$ in lesioned animals.

\section{Layer IV barrel neurons}

Receptive field size: $R S U$ s

Figure $3 A$ depicts sample PSTHs of a layer IV control RSU showing responses to the deflection of the principal whisker and four adjacent whiskers. This cell responded to the PW and showed minimal responses to two AWs. Figure $3 B$ shows sample PSTHs of a layer IV RSU from a lesioned rat. This cell responded to the PW and one AW. To assess the effects of brainstem lesions on receptive field size, we compared results from 37 RSUs from control animals and 39 RSUs from lesioned rats (Fig. 3C). In control animals, $37.8 \%$ of layer IV RSUs had receptive field sizes between four and five with $27.0 \%$ having receptive field sizes between one and two. In contrast, only $17.1 \%$ of layer IV RSUs in lesioned animals responded to four or five whiskers with $58.5 \%$ having receptive field sizes between one and two.

These changes were quantitatively compared by computing AW/PW ratios (as described above) for each cell. We first compared results from animals with midline lesions and those with lesions in SpVi. RSUs from animals with midline lesions had a mean AW/PW ratio that was indistinguishable ( $p=0.31$; Mann-Whitney $U$ test) from the ratio of RSUs recorded from animals with lesions in SpVi (Table 1). We therefore combined these results and compared them to $\mathrm{AW} / \mathrm{PW}$ ratios from control animals. Figure $3 D$ depicts box plots of the AW/PW ratios from control rats and from SpVi-lesioned rats. The mean AW/PW ratio for RSUs in control rats was $0.21 \pm 0.18$ (median, $0.17 ; n=$ 37 ), which was reduced significantly to $0.11 \pm 0.12$ (median, $0.09 ; n=39$ ) after the lesion ( $p=0.008)$.

\section{Receptive field size: FSUs}

Receptive fields of most layer IV FSUs are typically larger than those of RSUs (Simons and Carvell, 1989; Bruno and Simons, 2002). The PSTHs in Figure $4 \mathrm{~A}$ show responses of a control FSU to deflection of the PW and four AWs, demonstrating that this cell had a receptive field size of five. The set of PSTHs in Figure $4 B$ show responses typical of an FSU after SpVi lesions: responses to AWs are greatly reduced, with this cell having a receptive field size of three. The histogram in Figure $4 C$ was computed from data from 28 cells from control rats and 13 cells from rats with lesions. Note the shift in the distribution from relatively large receptive fields to smaller receptive field sizes after the lesion. Under control conditions, $60.0 \%$ of FSUs had receptive field sizes between four and five with only $20.0 \%$ having receptive field sizes between one and two. In contrast, after the lesions only $30.8 \%$ had receptive field sizes between four and five with $46.2 \%$ having receptive field sizes between one and two. We quantified these changes by computing AW/PW ratios, as described above. In control animals, FSUs had a mean ratio of $0.35 \pm 0.22$ (median, $0.29 ; n=28$ ). The AW/PW ratio was significantly smaller $(p=0.001)$ in FSUs from lesioned rats $(0.12 \pm$ 0.11 ; median, $0.07 ; n=13$ ) (Fig. $4 D$ ). [We combined data from both types of lesions because they produced statistically indistinguishable AW/PW ratios (Table 1).]

\section{Response kinetics}

Brainstem lesions offer an opportunity to assess the role of direct thalamocortical inputs in determining other properties of corti- 
cal responses to whisker stimuli. Figure $5 A$ depicts boxcar plots showing the distribution of response magnitudes for RSUs and FSUs recorded from control and lesioned animals to deflection of the PW. Response magnitude was computed as the mean number of significant spikes per stimulus within a $20 \mathrm{~ms}$ window after whisker deflection (averaged across all eight deflection angles). We first determined that response magnitudes were statistically indistinguishable after midline or nuclear lesions (Table 1) and therefore combined the data obtained using the two lesion paradigms. Control RSUs had a response magnitude of $0.74 \pm 0.62$ spikes per stimulus (median, 0.53 ) that was similar to response magnitude of RSUs obtained from animals that sustained brainstem lesions (0.61 \pm 0.55 ; median, $0.38 ; p=0.23)$. In contrast, FSU response magnitudes were smaller in lesion animals $(2.68 \pm 1.60$ spikes per stimulus, median, 2.64 vs $1.17 \pm 0.68$ spikes per stimulus, median, $1.33 ; p=0.009)$. The effects of brainstem lesions on response magnitudes, and other responses kinetics are depicted also in the population PSTH (Fig. 5C).

We defined the onset of $\mathrm{ON}$ responses as two consecutive, statistically significant PSTH bins and the end of each ON response as three consecutive bins with no significant activity. We combined data from animals with midline and nuclear lesions, because these were statistically indistinguishable (Table 1). RSUs from control animals had response duration (PWevoked response) of $34.46 \pm 25.50 \mathrm{~ms}$ (median, $31 \mathrm{~ms}$ ), whereas those from lesioned animals had mean response duration of $21.94 \pm 10.96 \mathrm{~ms}$ (median, $19 \mathrm{~ms}$ ). This difference was statistically significant $(p=0.004)$. FSU response duration did not change $(p=0.22)$ after brainstem lesions. FSUs in control animals had a mean response duration of $20.82 \pm 8.85$ ms (median, 18), whereas that obtained from animals that sustained brainstem lesion was $17.77 \pm 8.15$ (median, 17).

\section{OFF/ON ratios}

Next we tested for changes in OFF/ON ratios of both RSUs and FSUs. These ratios compare the magnitude of the stereotypical phasic responses to the onset and offset of the stimulus ramp evoked in response to PW deflection (Fig. 2A) (Simons, 1985). The boxcar plots in Figure $5 B$ depict the distribution in OFF/ON response ratios in both RSUs and FSUs. Ratios for RSUs in controls were $0.76 \pm 0.32$ (median, 0.73), whereas RSUs from brainstem-lesioned animals had a ratio of $1.43 \pm 1.83$ (median, $0.96)$; this difference was statistically significant $(p=0.003)$. Similarly, OFF/ON ratios for FSUs were significantly $(p=0.03)$ affected by the lesions; control FSUs had a ratio of $0.71 \pm 0.29$ (median, 0.77) that increased to $0.99 \pm 0.41$ (median, 0.96). By comparison, OFF/ON ratios in VPM were not affected significantly by brainstem lesions ( $0.42 \pm 0.45$, median, 0.29 in control animals vs $0.72 \pm 0.70$, median, 0.63 in animals that sustained brainstem lesion; $p=0.17$ ) (Fig. $5 B$ ).
B

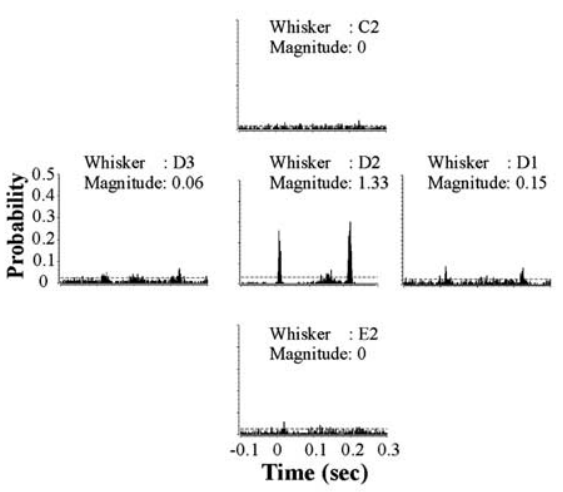

D

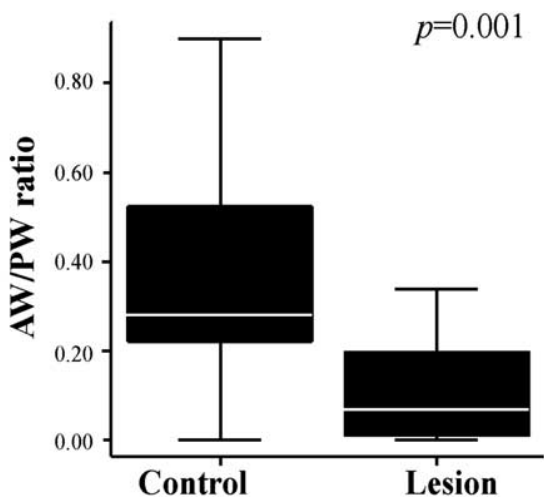

Figure 4. Representative PSTHs showing responses of a control FSU $(\boldsymbol{A})$ and an FSU from an animal that sustained a brainstem ratios of FSUs in control animals and animals with brainstem lesions. There is a significant ( $p=0.001$; Mann-Whitney $U$ test) reduction in AW/PW ratios after brainstem lesions. "Whiskers" are as described in the legend to Figure 2.

Angular selectivity

Barrel cortex neurons are selective for the angle of whisker deflection, eliciting larger magnitude responses to preferred angle deflections (Simons, 1985). Figure 6, $A$ and $B$, shows PSTHs for two cells of different angular tuning, Figure $6 \mathrm{~A}$ depicting a poorly tuned cell and Figure $6 B$ a well tuned neuron. Angular tuning was computed for PW-evoked response only. The PSTHs were constructed from responses of the cell to deflections in the angles indicated, with $0^{\circ}$ representing deflection in the caudal direction and $90^{\circ}$ deflection in the dorsal direction. Polar plots were constructed by plotting the normalized (to the maximally activating angle) $\mathrm{ON}$ response magnitude in each direction against the direction of deflection. To quantify angular preferences, we determined, for each neuron, the number of deflection angles evoking an $\mathrm{ON}$ response magnitude that was statistically different from responses to the maximally activating angle (compared using a one-tailed Student's $t$ test; $p<0.05$ ). We then categorized cells into eight groups $(0-7)$ representing the number of angles with responses that are statistically smaller than responses to the maximally activating angle. Category 0 represents the least-tuned cells (cells that respond equally to all deflection angles) (Fig. 6A), and category 7 represents the best-tuned cells (cells that respond preferentially to one deflection angle). For example, the cell depicted in Figure $6 B$ had an angular 
A

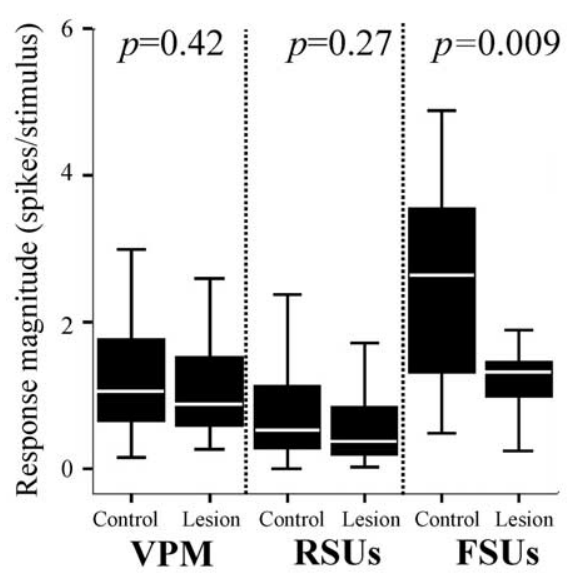

B

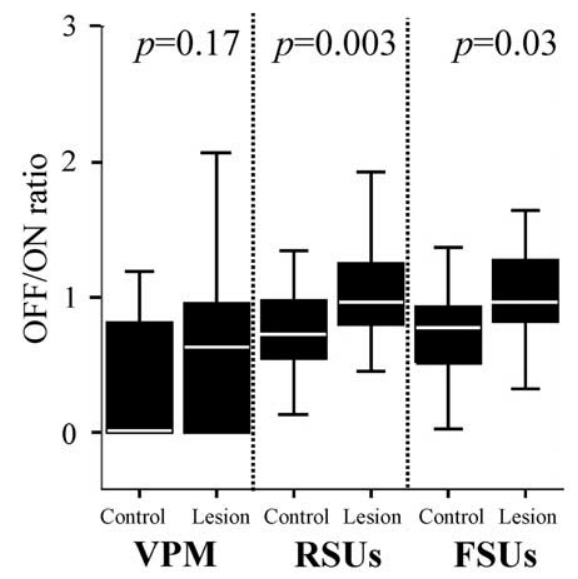

$2.7 \pm 1.8$ spikes/s (median, 2.6) in animals with brainstem lesions.

Infragranular neurons

We investigated also the effects of brainstem lesions on neurons recorded in layer V. We compared results from 11 RSUs from control animals to 15 RSUs from animals with lesions, focusing on the same parameters examined for layer IV neurons (see above). The receptive field size, assessed with the AW/PW ratio, did not change after the lesions. Although there were small increases in response magnitude and OFF/ON ratios after lesions, these did not reach statistical significance (Table 1). Response duration was decreased somewhat after the lesion, but the large variation in response duration of cells from control animals resulted in there being no significant difference between the two populations. Thus, in contrast to their layer IV counterparts, we found no significant differences in the response properties of infragranular neurons after brainstem lesions.

\section{Discussion}

Our aim was to assess the role of thalamic inputs in establishing the response properties of barrel cortex neurons. We reasoned that if AW responses are determined by thalamic inputs, brainstem lesions that suppress AW responses of thalamic neurons would also suppress these responses in barrel neurons. In contrast, if AW responses were created via intracortical interactions, the lesions would not affect receptive field sizes. Our results support the conclusion that AW responses in layer IV barrels are established, in large part, by direct thalamic inputs.

Figure 5. A, Boxplots showing the distribution of response magnitude of RSUs, FSUs, and VPM neurons in control and in animals with lesions. Brainstem lesions resulted in a significant decrease in the response magnitude of FSUs but not of RSUs or VPM neurons. $\boldsymbol{B}$, The $0 \mathrm{FF} / \mathrm{ON}$ ratios of RSUs increased significantly after the lesions. There was a similar increase in $0 \mathrm{FF} / \mathrm{ON}$ ratios of FSUs after brainstem lesions. In contrast, the increase in OFF/ON ratios of VPM neurons was not statistically significant. "Whiskers" are as described in the legend to Figure 2. C, Population PSTHs showing the response of RSUs (top row) and FSUs (bottom row) to PW and AW deflection in control animals and animals that sustained brainstem lesions (Lesion). The number of neurons ( $n$ ) used to construct each PSTH is indicated. The horizontal line represents the $99 \%$ confidence interval.

\section{Receptive field sizes}

Brainstem lesions resulted in significant reductions in the receptive field size of both RSUs and FSUs. After the lesions, the mean AW/PW response magnitude ratio

tuning category of 5 . We then pooled data on angular tuning categories from 39 RSUs in control animals and 39 RSUs in lesioned animals to construct the distribution histogram shown in Figure 6C. Of the control cells, 30.7\% were well tuned (having tuning ratios between 5 and 7 ), whereas $28.2 \%$ neurons from lesioned animals were well tuned. This difference was not statistically significant $\left(\chi^{2}=3.28 ; \mathrm{df}=7 ; p \leq 1\right)$. We conclude that brainstem lesions do not significantly affect the angular tuning of layer IV barrel neurons.

\section{Spontaneous activity}

Spontaneous firing rates of RSUs decreased significantly $(p=$ 0.03 ) from $3.1 \pm 3.5 \mathrm{~Hz}$ (median, 2.3) in control animals to $1.9 \pm$ $2.4 \mathrm{~Hz}$ (median, 0.8) in animals with brainstem lesions. Similarly, the spontaneous activity of FSUs decreased significantly $(p<$ 0.001 ) from $14.3 \pm 9.3 \mathrm{~Hz}$ (median, 13.7) in control animals to of RSUs decreased by $47.6 \%$. FSUs, which normally have significantly larger receptive fields than RSUs (Simons and Carvell, 1989), were even more affected by the lesions: their AW/PW ratios decreased by $65.7 \%$. That lesion-induced reductions in thalamic receptive fields had a greater effect on FSUs is consistent with the hypothesis that FSU responses more linearly reflect the response properties of their presynaptic thalamocortical units (Simons and Carvell, 1989; Miller et al., 2001; Bruno and Simons, 2002).

Although the reductions in AW responses were significant, some neurons retained their AW responses after the lesions. The source of this residual AW response could be the subpopulation of VPM neurons that are resistant to these lesions (Fig. 2A) (Timofeeva et al., 2004). These residual responses may be the result of incomplete lesions of SpVi (Fig. 1) or of the internuclear 
A

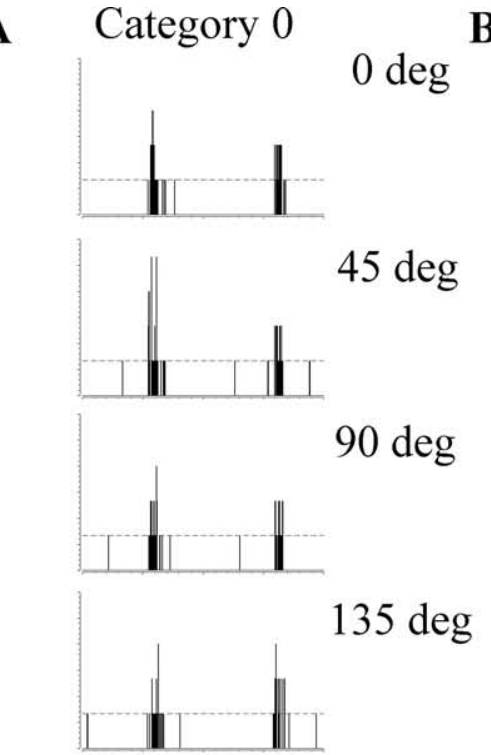

1.t.
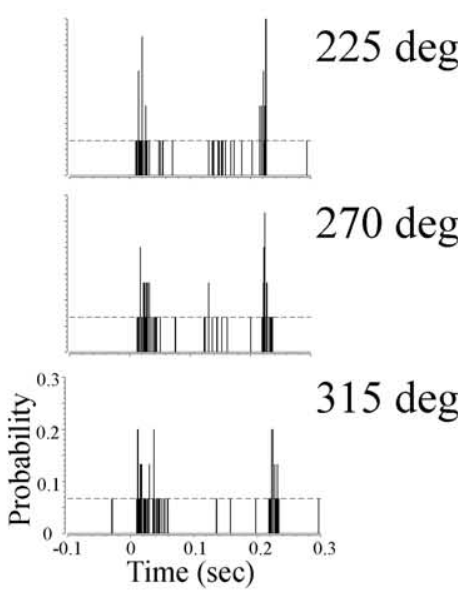

315 deg

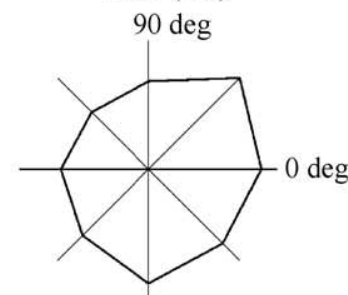

C

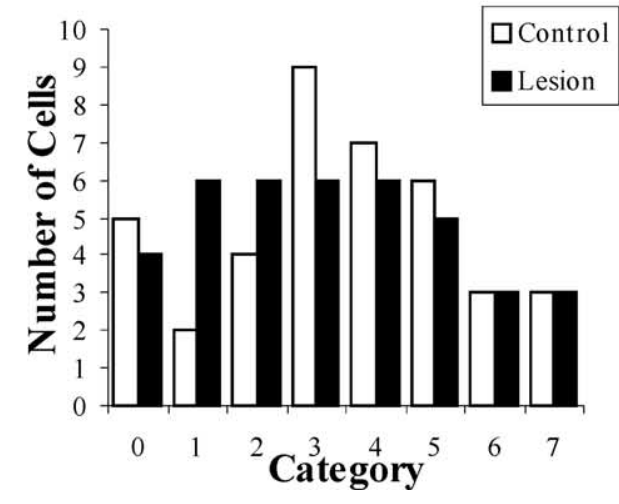

pathway between SpVi and PrV. It is possible, therefore, that more extensive lesions would have abolished all AW responses. Interestingly, the residual adjacent whisker responses were qualitatively similar to AW responses from control animals (Fig. 5C). An additional source for these residual AW responses may be the small population of thalamocortical axons that diverge to and terminate in two adjacent barrels (Land et al., 1995; Keller and Carlson, 1999; Arnold et al., 2001).

RSU receptive fields are normally constrained by feedforward inhibition from FSUs (Pinto et al., 2000, 2003), such that pharmacological suppression of this intracortical inhibition can unmask AW responses (Kyriazi et al., 1996). Here, the magnitude of feedforward inhibition was presumably reduced after brainstem lesions (Fig. 5A) (see below), as evidenced by lower response magnitudes of inhibitory neurons. AW/PW ratios did not, however, increase correspondingly. This suggests that AW responses are not relayed to the barrels in lesioned animals and supports the hypothesis that AW responses are normally conveyed by thalamocortical inputs.

Alternatively, AW responses resistant to the lesions may be generated through interbarrel interactions. However, anatomical data show sparse intracortical connections between barrels (Woolsey et al., 1975; Simons and Woolsey, 1979; Hoeflinger et al., 1995; Feldmeyer et al., 1999; Keller and Carlson, 1999) and that many of these link neurons in barrel septa (Kim and Ebner, 1999). Sparse intercolumnar connections also target the supragranular branches of apical dendrites belonging to layer IV star pyramids (Schubert et al., 2003), and these mediate weak synaptic interactions (Feldmeyer et al., 1999; Petersen and Sakmann, 2000; Schubert et al., 2003). In addition, analyses of voltagesensitive dye signals fail to demonstrate direct interbarrel pathways (Laaris et al., 2000; Laaris and Keller, 2002; Petersen et al., 2003). Finally, under the light narcosis used here, AW responses are unaffected by lesions of adjacent barrels (Goldreich et al., 1999). Thus, the preponderance of converging evidence suggests that intracortical interactions have a minor role in generating AW responses in barrel neurons.

In contrast to the findings by Goldreich et al. (1999), Armstrong-James et al. (1991) reported that AW responses are suppressed by lesions of adjacent barrels (Fox, 1994). We have argued previously that these results may reflect the use of the anesthetic urethane (Simons et al., 1992). Fox et al. (2003) reported that under either urethane or pentobarbital, pharmacological suppression of intracortical circuits reduces AW responses in layer IV barrels. Both thalamocortical and intercolumnar transmission could be enhanced during periods of reduced thalamic background activity that accompanies general anesthesia (Wan et al., 2003), provided that the level of anesthesia is not so deep as to substantially reduce cortical excitability. Reduced VPM spontaneous activity likely leads to a diminution of cortical feedforward inhibition (Bruno and Simons, 2002) and less short-term depression at thalamocortical synapses (CastroAlamancos and Oldford, 2002). Both effects would disproportionately increase weaker responses (Kyriazi et al., 1996) and thus

Figure 6. PSTHs of responses of a poorly tuned (category $0 ; \boldsymbol{A}$ ) and a well tuned (category 5 ; $\boldsymbol{B}$ ) neuron to deflection of a single whisker in eight different directions. Polar plots (below the histograms) were constructed by plotting the normalized response magnitude against the direction of whisker deflection. $0^{\circ}$ represents deflection in the caudal direction, and $90^{\circ}$ is deflection in the dorsal direction. The horizontal dotted line represents the $99 \%$ confidence interval. Stimulus onset is at $t=0$, and stimulus offset at $t=200 \mathrm{~ms}$. deg, Degrees. C, A histogram showing the distribution of angular selectivity of control RSU and RSUs obtained from animals with brainstem lesions (Lesion). 
increase AW/PW ratios (Kelly et al., 1999; Pinto et al., 2003; Castro-Alamancos, 2004). Indeed, under urethane, AW responses are substantially reduced during "arousal" (and thalamic activation) produced by electrical stimulation of the reticular formation (Castro-Alamancos, 2002). Under urethane or pentobarbital, where thalamic AW responses are small, cortical AW responses are unusually large and could well be mediated by normally weak intercolumnar connections that are otherwise mostly ineffective during information processing states. Indeed, the AW/PW ratio is approximately twofold greater under urethane anesthesia than under fentanyl analgesia (Simons et al., 1992) as a result of the unusually large AW responses. In this regard, it is noteworthy that Fox et al. (2003) recorded only from RSUs, which have the smallest receptive fields and were less impacted by brainstem lesions, compared with FSUs. It is therefore possible that these RSUs included star pyramids that receive intercolumnar inputs from adjacent barrel columns, inputs that may contribute to their AWs (Schubert et al., 2003).

\section{Response magnitude}

We were concerned that brainstem lesions might reduce the PW response magnitude of layer IV RSUs, thereby compromising putative intracortical excitatory connections that may contribute to AW responses. We also considered the possibility that the lesion-induced reduction in spontaneous activity of VPM and cortical neurons may reflect a reduced excitability of these cells, further compromising putative intercolumnar interactions. However, the lesions did not affect the response magnitude of either thalamic neurons or RSUs to their PW (Fig. 5A). Furthermore, AW responses of layer $\mathrm{V}$ neurons were unaffected by these same lesions. We conclude that the reduction in AW responses reflects a reduction in the AW responses of their presynaptic VPM neurons and not a nonspecific effect of brainstem lesions on cortical responsiveness.

In contrast, the response magnitude of FSUs to their PW was significantly reduced after the lesions. These findings suggest that FSUs may receive inputs from a specific population of thalamic neurons, the PW responses of which are dependent on inputs from SpVi. This possibility is supported by the finding that FSUs, unlike RSUs, receive inputs from the most strongly responsive VPM neurons (Bruno and Simons, 2002).

$\mathrm{RSU}$ responses to stimulus offset are weaker than to stimulus onset, in part because of feedforward and local inhibition (Kyriazi et al., 1994). Thus, OFF/ON ratios are an indirect metric for the efficacy of intrabarrel inhibition. Here, the decrease in inhibition was evidenced by the decrease in the response magnitude and spontaneous firing of FSUs. Furthermore, OFF/ON ratios of RSUs were significantly increased after brainstem lesions (Fig. 5B). As discussed above, despite the apparent reduction in intrabarrel inhibition, AW responses were not unmasked, further supporting the hypothesis that these responses are determined by direct thalamic inputs. The increase in the OFF response may also reflect increased low-threshold bursting in thalamic cells (Kyriazi et al., 1994), a result of lesion-induced reduction in spontaneous activity in these neurons.

\section{Angular selectivity}

Pharmacological suppression of inhibition in barrels leads to a reduction in angular tuning of RSUs (Kyriazi et al., 1996). This is thought to occur through GABAergic effects on tonic or shunting inhibition (Kyriazi et al., 1996), similar to the role of inhibition in shaping orientation selectivity of visual cortex neurons (Nelson et al., 1994). The fact that brainstem lesions had no significant effect on angular selectivity, despite the apparent reduction in intrabarrel inhibition, is consistent with the hypothesis that angular tuning of barrel neurons is strongly dependent on thalamic inputs that have an angular selectivity that remains unaffected by brainstem lesions.

\section{Infragranular layers}

Brainstem lesions had no effect on responses of layer $V$ neurons. This suggests that the response properties of infragranular neurons, including their AW response, are mediated by intracortical interactions (Simons, 1978; Armstrong-James and Fox, 1987). The dense and widespread intracolumnar and intercolumnar synaptic pathways are a likely substrate for these interactions (Keller, 1989; Bernardo et al., 1990; Gottlieb and Keller, 1997; Feldmeyer and Sakmann, 2000; Lubke et al., 2000; Laaris and Keller, 2002).

In conclusion, the results of this study support the hypothesis that AW response in the layer IV barrels is generated primarily by direct thalamocortical inputs. Brainstem lesions that abolish the AW response of thalamocortical neurons resulted in significant suppression of AW response in both inhibitory and excitatory barrel neurons. This effect was most prominent in inhibitory neurons, consistent with the hypothesis that the response properties of these cells more faithfully reflect the responses of their presynaptic thalamocortical afferents (Bruno and Simons, 2002).

\section{References}

Armstrong-James M (1995) The nature and plasticity of sensory processing within adult rat barrel cortex. In: Cerebral cortex, Vol 11, The barrel cortex of rodents (Jones EG, Diamond IT, eds), pp 333-373. New York: Plenum.

Armstrong-James M, Callahan CA (1991) Thalamo-cortical processing of vibrissal information in the rat. II. Spatiotemporal convergence in the thalamic ventroposterior medial nucleus (VPm) and its relevance to generation of receptive fields of S1 cortical "barrel” neurones. J Comp Neurol 303:211-224.

Armstrong-James M, Fox K (1987) Spatiotemporal convergence and divergence in the rat SI barrel cortex. J Comp Neurol 263:265-281.

Armstrong-James M, Callahan CA, Friedman MA (1991) Thalamo-cortical processing of vibrissal information in the rat. I. Intracortical origins of surround but not centre-receptive fields of layer IV neurones in the rat S1 barrel field cortex. J Comp Neurol 303:193-210.

Arnold PB, Li CX, Waters RS (2001) Thalamocortical arbors extend beyond single cortical barrels: an in vivo intracellular tracing study in rat. Exp Brain Res 136:152-168.

Bernardo KL, McCasland JS, Woolsey TA, Strominger RN (1990) Local intra- and interlaminar connections in mouse barrel cortex. J Comp Neurol 291:231-255

Bruno RM, Simons DJ (2002) Feedforward mechanisms of excitatory and inhibitory cortical receptive fields. J Neurosci 22:10966-10975.

Castro-Alamancos MA (2002) Role of thalamocortical sensory suppression during arousal: focusing sensory inputs in neocortex. J Neurosci 22:9651-9655.

Castro-Alamancos MA (2004) Dynamics of sensory thalamocortical synaptic networks during information processing states. Prog Neurobiol 74:213-247.

Castro-Alamancos MA, Oldford E (2002) Cortical sensory suppression during arousal is due to the activity-dependent depression of thalamocortical synapses. J Physiol (Lond) 541:319-331.

Dalva MB, Weliky M, Katz LC (1997) Relationships between local synaptic connections and orientation domains in primary visual cortex. Neuron 19:871-880.

Douglas RJ, Koch C, Mahowald M, Martin KAC, Suarez HH (1995) Recurrent excitation in neocortical circuits. Science 269:981-985.

Feldmeyer D, Sakmann B (2000) Synaptic efficacy and reliability of excitatory connections between the principal neurons of the input (layer 4) and output layer (layer 5) of the neocortex. J Physiol (Lond) 525:31-39.

Feldmeyer D, Egger V, Lubke J, Sakmann B (1999) Reliable synaptic connections between pairs of excitatory layer 4 neurones within a single 'bar- 
rel' of developing rat somatosensory cortex. J Physiol (Lond) 521:169-190.

Ferster D, Koch C (1987) Neuronal connections underlying orientation selectivity in cat visual cortex. Trends Neurosci 10:487-493.

Fox K (1994) The cortical component of experience-dependent synaptic plasticity in the rat barrel cortex. J Neurosci 14:7665-7679.

Fox K, Wright N, Wallace H, Glazewski S (2003) The origin of cortical surround receptive fields studied in the barrel cortex. J Neurosci 23:8380-8391.

Friedberg MH, Lee SM, Ebner FF (2004) The contribution of the principal and spinal trigeminal nuclei to the receptive field properties of thalamic VPM neurons in the rat. J Neurocytol 33:75-85.

Goldreich D, Kyriazi HT, Simons D (1999) Functional independence of layer IV barrels in rodent somatosensory cortex. J Neurophysiol 82:1311-1316.

Gottlieb JP, Keller A (1997) Intrinsic circuitry and physiological properties of pyramidal neurons in rat barrel cortex. Exp Brain Res 115:47-60.

Hoeflinger BF, Bennett-Clarke CA, Chiaia NL, Killackey HP, Rhoades RW (1995) Patterning of local intracortical projections within the vibrissae representation of rat primary somatosensory cortex. J Comp Neurol 354:551-563.

Hubel DH, Wiesel TN (1962) Receptive fields, binocular interaction and functional architecture in the cat's visual cortex. J Physiol (Lond) 160:106-154.

Hubel DH, Wiesel TN (1968) Receptive fields and functional architecture of monkey striate cortex. J Physiol (Lond) 195:215-243.

Keller A (1989) Functional properties of cortical neurons. In: Cortical circuits: synaptic organization of the cerebral cortex-structure, function and theory (White EL, ed), pp 107-131. Boston: Birkhäuser.

Keller A, Carlson GC (1999) Neonatal whisker clipping alters intracortical, but not thalamocortical projections in rat barrel cortex. J Comp Neurol 412:83-94.

Kelly MK, Carvell GE, Kodger JM, Simons DJ (1999) Sensory loss by selected whisker removal produces immediate disinhibition in the somatosensory cortex of behaving rats. J Neurosci 19:9117-9125.

Kim U, Ebner FF (1999) Barrels and septa: separate circuits in rat barrel field cortex. J Comp Neurol 408:489-505.

Kwegyir-Afful EE, Keller A (2004) Response properties of whisker related neurons in rat second somatosensory cortex. J Neurophysiol 92:2083-2092.

Kwegyir-Afful EE, Bruno RM, Simons DJ, Keller A (2004) The role of thalamic inputs in shaping surround receptive fields of barrel neurons. Soc Neurosci Abstr 30:978.971.

Kyriazi HT, Carvell GE, Simons DJ (1994) OFF response transformations in the whisker/barrel system. J Neurophysiol 72:392-401.

Kyriazi HT, Carvell GE, Brumberg JC, Simons DJ (1996) Quantitative effects of GABA and bicuculline methiodide on receptive field properties of neurons in real and simulated barrels. J Neurophysiol 75:547-560.

Laaris N, Keller A (2002) Functional independence of layer IV barrels. J Neurophysiol 87:1028-1034.

Laaris N, Carlson GC, Keller A (2000) Thalamic-evoked synaptic interactions in barrel cortex revealed by optical imaging. J Neurosci 20:1529-1537.

Land PW, Buffer SA, Yaskosky JD (1995) Barreloids in adult rat thalamus: three-dimensional architecture and relationship to somatosensory cortical barrels. J Comp Neurol 355:573-588.

Lubke J, Egger V, Sakmann B, Feldmeyer D (2000) Columnar organization of dendrites and axons of single and synaptically coupled excitatory spiny neurons in layer 4 of the rat barrel cortex. J Neurosci 20:5300-5311.

Miller KD, Pinto DJ, Simons DJ (2001) Processing in layer 4 of the neocortical circuit: new insights from visual and somatosensory cortex. Curr Opin Neurobiol 11:488-497.
Minnery BS, Simons DJ (2003) Response properties of whisker-associated trigeminothalamic neurons in rat nucleus principalis. J Neurophysiol $89: 40-56$.

Mountcastle VB, Powell TPS (1959) Neural mechanisms subserving cutaneous sensibility, with special reference to the role of inhibition in sensory perception and discrimination. Bull Johns Hopkins Hosp 105:201-232.

Mountcastle VB, Davies PW, Berman AL (1957) Response properties of neurons of cat's somatic sensory cortex to peripheral stimuli. J Neurophysiol 20:374-407.

Nelson S, Toth L, Sheth B, Sur M (1994) Orientation-selectivity of cortical neurons during intracellular blockade of inhibition. Science 265:774-777.

Petersen CCH, Sakmann B (2000) The excitatory neuronal network of rat layer 4 barrel cortex. J Neurosci 20:7579-7586.

Petersen CCH, Grinvald A, Sakmann B (2003) Spatiotemporal dynamics of sensory responses in layer $2 / 3$ of rat barrel cortex measured in vivo by voltage-sensitive dye imaging combined with whole-cell voltage recordings and neuron reconstructions. J Neurosci 23:1298-1309.

Pinto DJ, Brumberg JC, Simons DJ (2000) Circuit dynamics and coding strategies in rodent somatosensory cortex. J Neurophysiol 83:1158-1166.

Pinto DJ, Hartings JA, Simons DJ (2003) Cortical damping: analysis of thalamocortical response transformations in rodent barrel cortex. Cereb Cortex 13:33-44.

Rhoades RW, Belford GR, Killackey HP (1987) Receptive-field properties of rat ventral posterior medial neurons before and after selective kainic acid lesions of the trigeminal brain stem complex. J Neurophysiol 57:1577-1600.

Schubert D, Kotter R, Zilles K, Luhmann HJ, Staiger JF (2003) Cell typespecific circuits of cortical layer IV spiny neurons. J Neurosci 23:2961-2970.

Simons DJ (1978) Response properties of vibrissa units in rat SI somatosensory neocortex. J Neurophysiol 41:798-820.

Simons DJ (1985) Temporal and spatial integration in the rat SI vibrissa cortex. J Neurophysiol 54:615-635.

Simons DJ (1995) Neuronal integration in the somatosensory whisker/barrel cortex. In: Cerebral cortex, Vol 11, The barrel cortex of rodents (Jones EG, Diamond IT, eds), pp 263-297. New York: Plenum.

Simons DJ, Carvell GE (1989) Thalamocortical response transformation in rat vibrissa/barrel system. J Neurophysiol 61:311-330.

Simons DJ, Woolsey TA (1979) Functional organization in mouse barrel cortex. Brain Res 165:327-332.

Simons DJ, Carvell GE, Hershey AE, Bryant DP (1992) Responses of barrel cortex neurons in awake rats and effects of urethane anesthesia. Exp Brain Res 91:259-272.

Timofeeva E, Lavallee P, Arsenault D, Deschênes M (2004) Synthesis of multiwhisker-receptive fields in subcortical stations of the vibrissa system. J Neurophysiol 91:1510-1515.

Wan X, Mathers DA, Puil E (2003) Pentobarbital modulates intrinsic and GABA-receptor conductances in thalamocortical inhibition. Neuroscience 121:947-958.

Wolf W, Hicks TP, Albus K (1986) The contribution of GABA-mediated inhibitory mechanisms to visual response properties of neurons in the kitten's striate cortex. J Neurosci 6:2779-2795.

Woolsey TA, Van der Loos H (1970) The structural organization of layer IV in the somatosensory region (SI) of mouse cerebral cortex. Brain Res 17:205-242.

Woolsey TA, Dierker MK, Wann DF (1975) Mouse SmI cortex: qualitative and quantitative classification of Golgi-impregnated barrel neurons. Proc Natl Acad Sci USA 72:2165-2169.

Worgotter F, Koch C (1991) A detailed model of the primary visual pathway in the cat-comparison of afferent excitatory and intracortical inhibitory connection schemes for orientation selectivity. J Neurosci 11:1959-1979. 